\title{
An Alternate Iterative Differential Evolution Algorithm for Parameter Identification of Chaotic Systems
}

\author{
Wanli Xiang, ${ }^{1,2}$ Xuelei Meng, ${ }^{2}$ and Meiqing $\mathrm{An}^{2}$ \\ ${ }^{1}$ Institute of Systems Engineering, Tianjin University, Tianjin 300072, China \\ ${ }^{2}$ School of Traffic \& Transportation, Lanzhou Jiaotong University, Lanzhou, Gansu 730070, China \\ Correspondence should be addressed to Wanli Xiang; xiangwl@tju.edu.cn
}

Received 16 April 2015; Accepted 4 August 2015

Academic Editor: Daniele Fournier-Prunaret

Copyright (c) 2015 Wanli Xiang et al. This is an open access article distributed under the Creative Commons Attribution License, which permits unrestricted use, distribution, and reproduction in any medium, provided the original work is properly cited.

\begin{abstract}
Parameter estimation of chaotic systems plays a key role for control and synchronization of chaotic systems. At first, the parameter estimation of chaotic systems is mathematically formulated as a global continuous optimization problem. Then through integrating two differential mutation strategies, an improved greedy selection mechanism and a population diversity balance scheme, an alternate iterative differential algorithm, called AIDE, is presented to solve the problem in this paper. Subsequently, experiments are tested on a set of cases of parameter estimation of chaotic systems and the results show that AIDE is better than or at least equal to $\mathrm{DE} / \mathrm{rand} / 1 /$ bin, $\mathrm{DE} / \mathrm{best} / 1 / \mathrm{bin}$, and other four well-known algorithms in all cases.
\end{abstract}

\section{Introduction}

In 1963, Lorenz first found the classical chaotic attractor during the process of simulating the change of atmosphere through a three-dimensional autonomous system [1]. After that, the chaos theory is studied in detail by many researchers. In particular, control and synchronization of chaotic systems have a promising prospect in various fields like information science, medicine, biology, engineering, and so on. However, the chaotic systems to be controlled usually have some unknown parameters. Thus, the parameter estimation problem becomes the first key issue for solving the control and synchronization of chaotic systems.

Recently, numerous researchers had given much attention to the parameter estimation of chaotic systems [2-18]. In particular, great achievements over the parameter estimation of chaotic systems have been obtained by intelligent algorithms recently. For example, Dai et al. [19] transformed the problem of parameter estimation of chaotic systems into a global optimization problem through designing a suitable objective function and solved the optimization problem using genetic algorithm. Likewise, Chang [3] employed the differential evolution (DE) algorithm to estimate the unknown parameters of Rossler's chaotic system. Next, Chang [4] proposed an improved differential evolution algorithm to estimate the unknown parameters of Chen and Lü systems. He et al. [16] employed particle swarm optimization (PSO) algorithm for solving the problem of parameter estimation of Lorenz system and found that PSO is better than genetic algorithm (GA). Gao and Tong [20] also proposed an improved particle swarm optimization algorithm to effectively estimate the unknown parameters of Lorenz system and Lorenz system with noise. Meanwhile, a novel chaotic ant swarm (CAS for short) algorithm was developed to estimate the unknown parameters of Logistic and Lorenz systems by Li et al. [5]. Later, Chang et al. [6] introduced an evolutionary programming (EP) algorithm to solve the problem of parameter estimation of the unified chaotic systems including Lorenz, Lü, and Chen systems. Furthermore, some problems of parameter estimation of chaotic systems with time delay or other characteristics were also solved by some intelligent optimization algorithms [7-9]. In order to increasingly improve the accuracy of parameter estimation of chaotic systems, there are still some researchers who have proposed some improved PSO [10, 11], improved DE [2], and hybrid evolutionary algorithms $[12-14,21]$ to identify the unknown parameters of chaotic systems. In particular, Wang et al. [14] skillfully hybridize Nelder-Mead Simplex Search (NM for short) and 
differential evolution (DE) to propose a hybrid algorithm, called NMDE. NMDE was successfully used to identify the unknown parameters of chaotic systems and obtained a better convergence performance.

In order to further improve the accuracy of the parameter estimation of chaotic systems, inspired by the existence of a few big evolution eras and small evolution eras in nature, we propose an alternate iterative differential evolution algorithm, in which two mutation strategies with different search abilities (exploration and exploitation) are employed to imitate the evolutionary behaviour of big evolution era and small evolution era, respectively.

The rest of the paper is organized as follows. In Section 2, the problem description over parameter estimation is described. In Section 3, the traditional differential evolution algorithm is briefly described. Subsequently, an alternate iterative differential evolution, called AIDE, is presented in detail in Section 4. Nextly, comprehensive experiments are conducted in Section 5 to validate the performance of AIDE. Finally, a conclusion is drawn in Section 6.

\section{Problem Description}

In this paper, the following $n$-dimensional chaotic system is considered:

$$
\dot{X}=F\left(X, X_{0}, \theta_{0}\right),
$$

where $X \in \Re^{n}$ represents the state vector of chaotic systems, $X_{0} \in \mathfrak{R}^{n}$ is an initial state, namely, the start point of evolution of chaotic systems, $\theta_{0} \in \mathfrak{R}^{D}$ is the real parameters values of chaotic systems, and $D$ is the number of the parameters $[14$, $16,21,22]$.

When estimating the unknown parameters of chaotic systems, often suppose that the structure of the systems is known in advance [12] and further suppose that all states of the systems can be measured. Thus, the estimated system can be described as follows:

$$
\dot{Y}=F\left(Y, X_{0}, \theta\right),
$$

where $Y \in \mathfrak{R}^{n}$ denotes a state vector of the estimated system and $\theta \in \mathfrak{R}^{D}$ is a parameter vector of the estimated system.

In this way, the problem of parameter estimation of chaotic systems is to find a set of suitable parameters of $\theta$ and make them very close to real values of $\theta_{0}$. The corresponding objective function is to minimize the error between the state vector $Y$ of the estimated system and the state vector $X$ of the original system. Accordingly, the problem can be converted into an optimization problem. Generally, its objective can be described by (3). The specific principle of parameter estimation of chaotic systems is shown in Figure 1:

$$
\min _{\theta} J=\frac{1}{M} \sum_{k=1}^{M}\left\|X_{k}-Y_{k}\right\|^{2}
$$

where $M$ represents the length of the sampled data for parameters estimation, $X_{k}$ and $Y_{k}$ denote the state vector of the original system and the estimated system at time $k$, respectively, and $\|\cdot\|$ represents the 2-norm or the Euclidean norm.

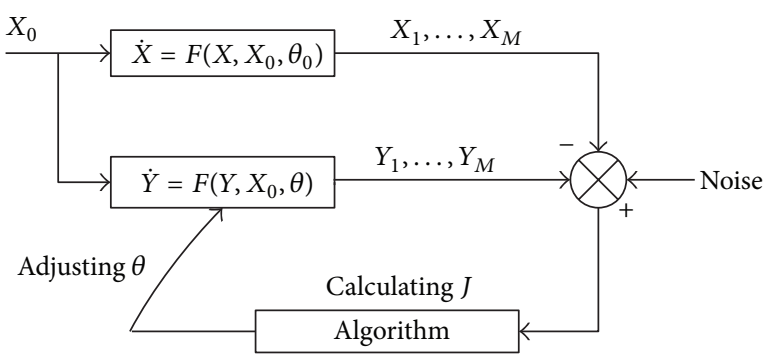

FIGURE 1: The principle of parameters estimation of chaotic systems.

\section{Canonical Differential Evolution}

In 1995, Storn and Price [23, 24] first proposed the differential evolution (DE) algorithm, which is simple yet powerful population-based and direct search algorithm. According to the mutation strategies and the crossover operators (bin and exp) of $\mathrm{DE}$ [24], it is very flexible and can be formed into many variants. In order to classify the different variants, the notation DE/ $x / y / z$ was introduced by Storn and Price [24]. Among a variety of variants, $\mathrm{DE} / \mathrm{best} / \mathrm{l} / \mathrm{bin}$ is one of the most popular variants and its exploitation ability is relatively good. To some extent, it may speed up the convergence speed of $\mathrm{DE} /$ best/1/bin. Like other population-based metaheuristics, $\mathrm{DE}$ also first randomly produces an initial population, which is composed of $N_{p} D$-dimensional vector, so-called individuals, which encode the candidate solutions; that is, $x_{i}=$ $\left(x_{i 1}, \ldots, x_{i D}\right), i=1, \ldots, N_{p}$. Thereafter, DE/best/1/bin enters a loop of evolutionary operations: mutation, crossover, and selection.

3.1. Mutation. For each target vector $x_{i}$, a mutant vector $v_{i}$ is generated according to the following equation:

$$
v_{i}=x_{\text {best }}+F \cdot\left(x_{a}-x_{b}\right),
$$

where $i=1,2, \ldots, N_{p}$, best is the index of the best individual with the best fitness value, $a, b \in\left[1, N_{p}\right]$ are mutually different random integer numbers and they are also different from current index $i$, and the scale factor $F$ is a real constant in the range of $[0,2]$ and it is used to control the amplification of the differential variation $\left(x_{a}-x_{b}\right)$ [24].

3.2. Crossover. At this phase, DE/best/1/bin employs a binomial crossover operator to generate a trial vector $u_{i}$ according to the following equation:

$$
u_{i j}= \begin{cases}v_{i j}, & \text { if rand }[0,1]_{j} \leqslant \mathrm{Cr} \vee j==j_{\text {rand }}, \\ x_{i j}, & \text { otherwise, }\end{cases}
$$

where $i=1,2, \ldots, N_{p}, j=1,2, \ldots, D, \operatorname{rand}[0,1]_{j}$ is a random real number between $[0,1]$, and $j_{\text {rand }} \in\{1,2, \ldots, D\}$ is a randomly chosen index to make sure that the trial vector $u_{i}$ gets at least one parameter from the mutant vector $v_{i}$. The crossover rate $\mathrm{Cr}$ is a predefined constant within the range $[0,1]$, which controls the fraction of parameter values copied from mutant vector [24]. 
3.3. Selection. After crossover operation, the trial vector $u_{i}$ is compared to the target vector $x_{i}$ using the greedy selection mechanism. To be specific, the selection process can be described by the following equation:

$$
x_{i}^{\star}= \begin{cases}u_{i}, & \text { if } f\left(u_{i}\right)<f\left(x_{i}\right), \\ x_{i}, & \text { otherwise, }\end{cases}
$$

where $f(x)$ denotes the objective of a solution $x$ and $x_{i}^{\star}$ represents a parent vector used to replace the target vector $x_{i}$ in the next generation.

\section{Alternate Iterative Differential Evolution}

4.1. Motivation. In view of pseudorandomness and sensitivity to initial state values of chaotic systems, all these chaotic behaviours may result in the objective function shown in (3) having complex landscape, which is the biggest challenge for parameter identification. Hence, better and robust evolutionary algorithm is needed to solve it.

In order to balance the exploration and exploitation ability of DE, two different mutation strategies with different searching abilities are employed in AIDE. Inspired by the existence of a few big evolution eras and small evolution eras in nature, together with the diversity of mutation strategy of DE, we use two mutation strategies with different searching abilities to simulate evolutionary behaviour of big evolution era and small evolution era, respectively. In other words, DE/best/1/bin with better exploitation ability is used to simulate the evolution behavior of big evolution era. Inspired by PSO, we presented a new mutation strategy, called $\mathrm{DE} / \mathrm{pbest} / 1 / \mathrm{bin}$, where pbest represents its own previous best individual for each target individual $x_{i}$. In this way, the exploration ability of $\mathrm{DE} /$ pbest/1/bin may be better than that of $\mathrm{DE} / \mathrm{best} / 1 /$ bin. For each target vector $x_{i}$, its own previous best individual instead of the global best one is used to guide the population evolution in DE/pbest/1/bin, which partially prevents population from rapidly clustering in the vicinity of the global best individual and avoids evolution stagnation. DE/pbest/1/bin is employed to simulate evolutionary behaviour of the small evolution era. Based on the analysis mentioned above, an alternate iterative differential evolution is presented to identify the unknown parameter of chaotic systems.

4.2. Population Initialization Based on Opposition-Based Learning. In order to further improve the performance of evolutionary algorithm, an opposition-based learning (OBL) technique proposed by Rahnamayan et al. $[25,26]$ is used to initialize a population. The specific procedure is given as the following steps.

Step 1. Randomly produce an initial population $P$ of $N_{p}$ individuals.

Step 2. Generate the opposite-population OP of $N_{p}$ opposite individuals according to OBL.
Step 3. Select $N_{p}$ fittest individuals from $P \bigcup O P$ as initial population.

4.3. Improved Greedy Selection Mechanism. Inspired by harmony search (HS) algorithm [27, 28], the selection mechanism of standard DE is changed according to the following criteria:

(i) In the canonical differential evolution, the greedy selection operation is carried out until the corresponding trial vector $u_{i}$ for each target vector $x_{i}$ ( $i=$ $\left.1,2, \ldots, N_{p}\right)$ is generated. In AIDE, the greedy selection operation is immediately conducted between the trial vector and the target vector when some trial vector is generated. In this way, the global best individual or the personal best individual (the previous best individual for each target vector) may be updated more rapidly than ever; namely, their new information helps to better guide the population to evolve towards better solutions.

(ii) Different from the canonical differential evolution, in AIDE, the new generated trial vector $u_{i}$ is first compared with the target vector $x_{i}$; if individual $u_{i}$ is not inferior to individual $x_{i}$, then $u_{i}$ will replace $x_{i}$, or else, the worst individual $x_{w}$ of current population is substituted with $u_{i}$ directly, which is taking a cue from the greedy selection scheme of HS. It should be noted that a greedy selection between new generated $u_{i}$ and $x_{w}$ is performed in HS. But here $u_{i}$ directly replaces $x_{w}$ in order to maintain a good population diversity.

In the presented AIDE algorithm, the improved greedy selection mechanism is employed.

4.4. Constraint Handling. When the $j$ th variable of the $i$ th individual $x_{i}$ violates the boundary constraints, the corresponding $j$ th component will be reset to a suitable value according to the following criterion:

$$
\begin{aligned}
& x_{i j} \\
& = \begin{cases}x_{j}^{\min }+\operatorname{rand}(0,1) \cdot\left(x_{j}^{\max }-x_{j}^{\min }\right), & \text { if } x_{i j}<x_{j}^{\min }, \\
x_{j}^{\max }-\operatorname{rand}(0,1) \cdot\left(x_{j}^{\max }-x_{j}^{\min }\right), & \text { if } x_{i j}>x_{j}^{\max } .\end{cases}
\end{aligned}
$$

4.5. Alternate Iterative Differential Evolution. According to the aforementioned analysis, two mutation strategies are employed in AIDE. One is DE/best/1, which is given in (4). Inspired by PSO, a new mutation strategy, called DE/pbest/1, is presented in AIDE. The mutation strategy DE/pbest/1 can be described as follows:

$$
v=x_{i}^{\text {pbest }}+F \cdot\left(x_{a}-x_{b}\right)
$$

where pbest is similar to that of PSO and denotes the index of personal best individual for each target vector $x_{i}$ and the other variables are the same as those of (4).

From (4), the first term of the equation is the global best individual, which means that the population evolves 
under the guidance of the global best individual and it owns extremely strong exploitation ability. The search equation is used to simulate the evolution behavior of big evolution era in nature. In big evolution era, species in nature evolve very rapidly from the low level to high level, from simplicity to complexity, and from barbarity to civilization.

From (8), the first term of the equation is the personal best individual of the corresponding target vector, which means that the population will be directed by the previous best one of each individual rather than the global best individual. Under such circumstance, the evolution speed of population slows down in contrast to the situation of guidance of (4). Equation (8) is used to simulate the evolution behaviour of the small evolution era in nature, which helps to further prevent the population from clustering around the global best individual. It should be noted that species in nature evolve slowly over a period of time due to the effects of environmental change, natural disaster, and so on.

As far as the time periods of the big evolution era and the small evolution era are concerned, two constant numbers, $\operatorname{limit} A$ and $\operatorname{limit} B$, are introduced to denote them. Namely, the DE/pbest/1/bin is carried out limit $B$ times after the $\mathrm{DE} /$ best/1/bin is performed $\operatorname{limit} A$ times; then the two procedures are run alternately. Generally, the value of limit $A$ is bigger than that of limit $B$.

In order to further improve the diversity of AIDE, inspired by the scout bees operation of artificial bee colony (ABC) algorithm proposed by Karaboga and Basturk [29], changed scout bees operation is introduced into AIDE. To be specific, if the global best individual is not further improved through a predetermined number of generations called limit, then the first top $(1 / 5) N_{p}$ individuals are replaced by randomly generated new individuals in order to maintain the population diversity and avoid the stagnation of evolution.

According to the above improvements, the main procedure of presented hybrid algorithm AIDE can be summarized in Algorithm 1.

\section{Experimental Study and Discussion}

5.1. Typical Systems and Parameter Settings. In this section, comprehensive experiments are conducted to verify the effectiveness of AIDE. As a typical chaotic system, Lorenz system is taken as an example. The Lorenz system is mathematically formulated as follows:

$$
\begin{aligned}
& \dot{x}_{1}=a \cdot\left(x_{2}-x_{1}\right), \\
& \dot{x}_{2}=b \cdot x_{1}-x_{1} \cdot x_{3}-x_{2}, \\
& \dot{x}_{3}=x_{1} \cdot x_{2}-c \cdot x_{3},
\end{aligned}
$$

where $a=10, b=28$, and $c=8 / 3$ are the original parameters and the system displays a chaotic attractor as shown in Figure 2.

During the process of parameter estimation for Lorenz system, the fourth order Runge-Kutta algorithm is employed to obtain state vector in our simulation, where the step size $h$ is set to 0.01 and the length of samples is selected as $M=300$. At first, let Lorenz system evolve freely from a random initial

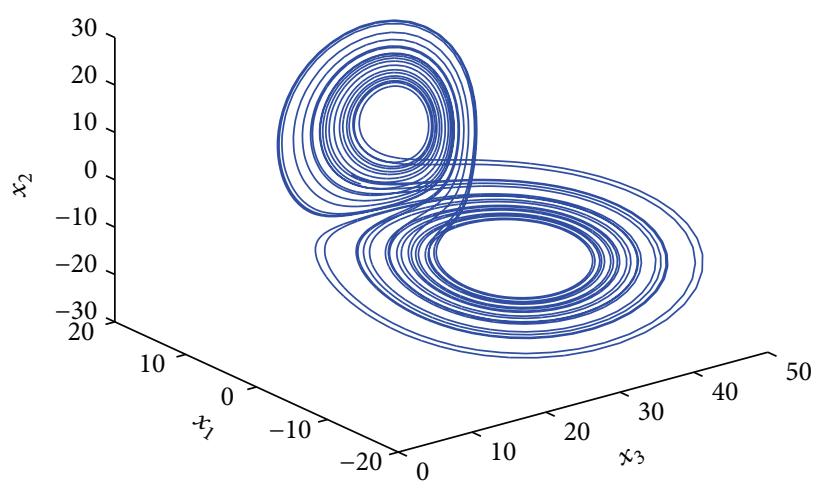

FIgURE 2: The chaotic attractor of Lorenz system.

state and choose any point as the initial state $X_{0}$ of system after a period of transient process. Next, successive $M$ state vectors of both the original system and the estimated system are used to calculate the objective $J$ as shown in (3). Last, the aim of minimizing the objective $J$ is to search a suitable set of parameters $\theta$.

For a fair comparison, the searching ranges are set as follows: $a \in[9,11], b \in[20,30]$, and $c \in[2,3]$, which are also used in $[14,16,21,22]$. In addition, the maximum cycle number MCN is taken as 100 , and the population size $N_{p}$ is set as 20,40 , and 120 when the number of unknown parameters is 1,2 , and 3, respectively, in the literature [14]. That is, the maximum number of function evaluations (maxFEs) is 2000 $(20 * 100), 4000(40 * 100)$, and $12000(120 * 100)$ when the number of unknown parameters is 1,2 , and 3 , respectively. That is, for fixed population size in AIDE, that is, $N_{p}=40$, the MCN is set as $50(2000 / 40), 100(4000 / 40)$, and 300 $(12000 / 40)$ when the number of unknown parameters is 1 , 2 , and 3 , respectively.

In order to further demonstrate the effectiveness of AIDE, it is first compared with DE/best/1/bin and DE/rand/1/bin. The population size $N_{p}$ is set to 40 for all cases of the three algorithms. The maximum cycle number of the three algorithms is the same as aforementioned. Some other common parameters settings of the three algorithms are also similar to each other; that is, $\mathrm{Cr}=0.9$ and $F=0.5$. What is more, in AIDE, the parameter limit is set to 10 , the parameter limit $A$ is set to 1000 , and the parameter $\operatorname{limit} B$ is set to 500 by trial and error.

\subsection{Comparison between AIDE, DE/best/1/bin, and $\mathrm{DE} / \mathrm{rand} / 1 / \mathrm{bin}$}

\subsubsection{Simulation on One-Dimensional Parameter Estimation.} First, experiments tested on three one-dimensional parameter estimation cases are conducted. That is, only one parameter among parameters $a, b$, and $c$ is unknown and needs to be identified at a time. In addition, each algorithm is run twenty times independently for each case, respectively. The statistical results obtained by $\mathrm{AIDE}, \mathrm{DE} / \mathrm{best} / 1 / \mathrm{bin}$, and $\mathrm{DE} / \mathrm{rand} / 1 / \mathrm{bin}$ for the three cases are listed in Table 1. Meanwhile, the evolution process of unknown parameters $(a, b$, and $c)$ and average of objective function values $J$ are plotted in Figure 3. 
(1) Initialize the population $X$ using opposition-based learning

(2) Let $X^{\text {pbest }}=\left\{x_{i}^{\text {pbest }}, i=1,2, \ldots, N_{p}\right\}$ represent the population composed of the best one of each individual $\left(x_{i}^{\text {pbest }}\right)$ in history

(3) Set iteration $=1 / /$ Iterative variable

(4) while iteration $\leqslant M C N$ do

(5) for $i=1$ to $N_{p}$ do

(6) Choose the best individual from the current population, and let best represent its index

(7) Generate two random integer numbers $a, b \in\left[1 \cdots N_{p}\right] \wedge a \neq b \neq i$

(8) if $\operatorname{true} A==0$ then

(9) Perform differential mutation according to (4)

(10) Set limit $A=$ limit $A-1$

(11) else

(12) Perform differential mutation according to (8)

(13) Set limitB $=$ limitB -1

(14) end if

(15) Do bound constraints handling according to (7)

(16) Generate trial vector $u$ according to (5) and compute its objective value

(17) if $f(u)$ is better than $f\left(x_{i}\right)$ then

(18) Replace $x_{i}$ with $u$ immediately

(19) else

(20) Substitute the worst individual $x_{w}$ in current population with $u$ immediately and $w$ is the index of the worst individual

(21) if $f(u)$ is better than $f\left(x_{w}^{\text {pbest }}\right)$ then

(30)

(31)

isaster mutation

(32) if limit $_{2}>$ limit then

(33) Sort the population according to objective value order by ascent and replace the first (1/5) $N_{p}$ individuals with randomly generated individuals

(34) Set limit $_{2}=0$

(35) end if

(36) Record the best solution found so far

(37) if the best solution is updated then

(38) ${\text { Set } \text { limit }_{2}=0}$

(39) else

(40) Set limit $2=$ limit $_{2}+1$

(41) end if

(42) Set iteration = iteration +1

(43) end while

Algorithm 1: The procedure of AIDE.

From Table 1, it can be seen that $\mathrm{DE} / \mathrm{rand} / 1 /$ bin is worse than both DE/best/1/bin and AIDE, which shows that the convergence speed of DE/rand/1/bin is slow. Furthermore, the objective values obtained by AIDE and DE/best/1/bin are all zero, which means that the estimated value of $a, b$, and $c$ identified by AIDE and DE/best/1/bin is exactly equal to the true value of the corresponding parameter, respectively. Nevertheless, the convergence speed of $\mathrm{DE} / \mathrm{best} / \mathrm{l} / \mathrm{bin}$ is slower than that of AIDE, which can be found from Figure 3, which indicates that the combination of the two mutation strategies is more favourable to speed up the convergence of AIDE than $\mathrm{DE} /$ best/1/bin itself. That is, sometimes the mutation strategy $\mathrm{DE} /$ pbest/1/bin may work better than DE/best/1bin.

5.2.2. Simulation on Two-Dimensional Parameter Estimation. Second, Experimental study about three two-dimensional parameter estimation cases is carried out. At this time, two of the three parameters are unknown and need to be identified. The corresponding statistical results are listed in Tables 2-4, respectively. The corresponding evolution processes of both 


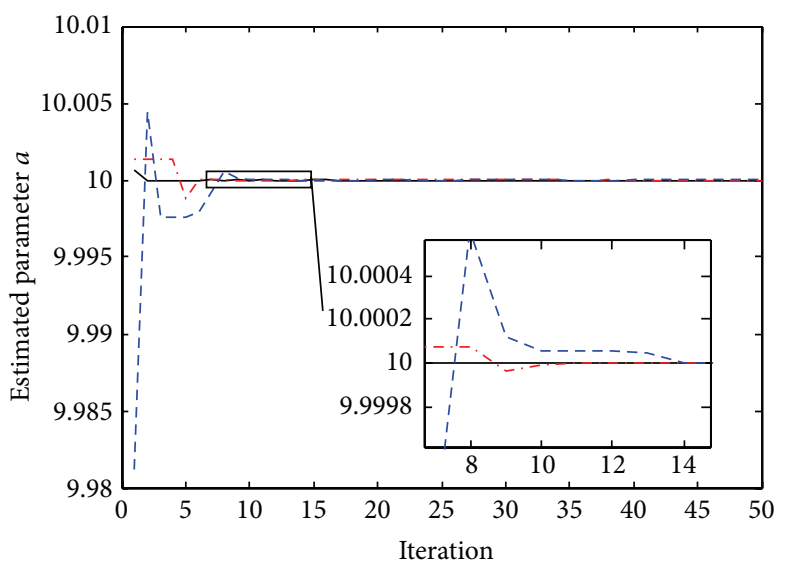

(a)

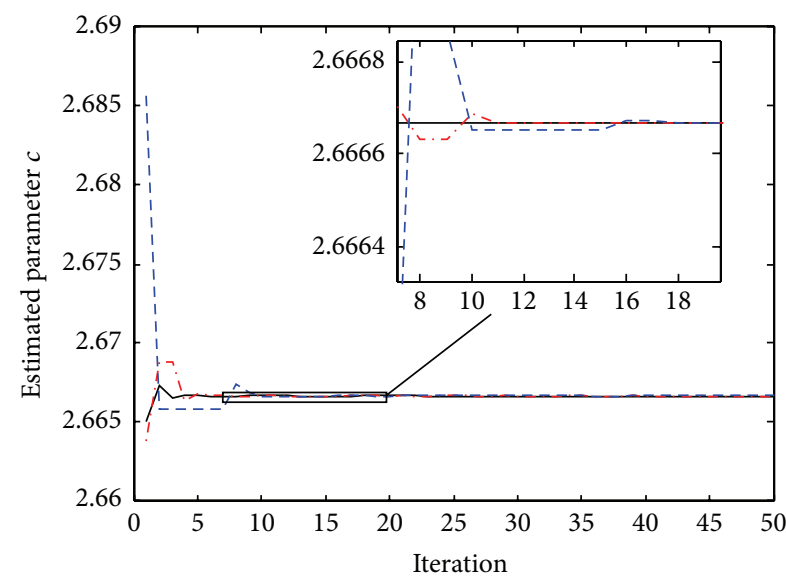

(c)

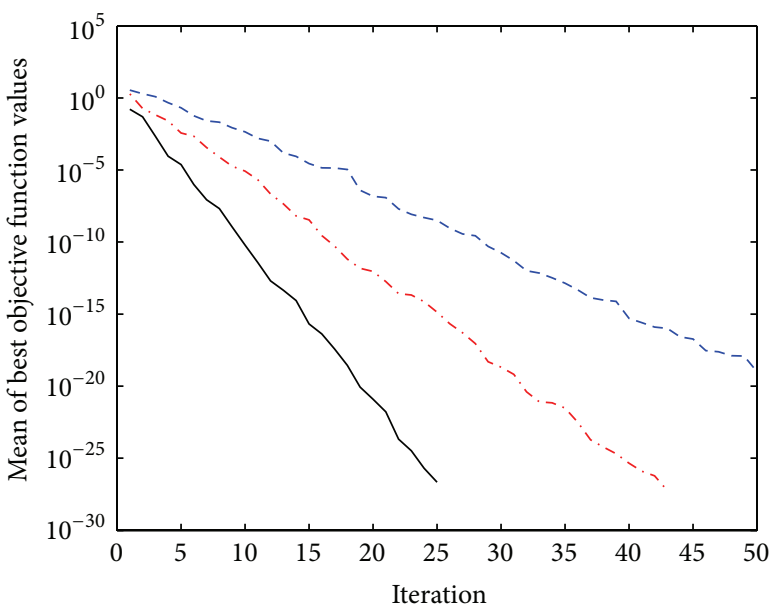

- AIDE

...- DE/best/1/bin

- - DE/rand/1/bin

(e)

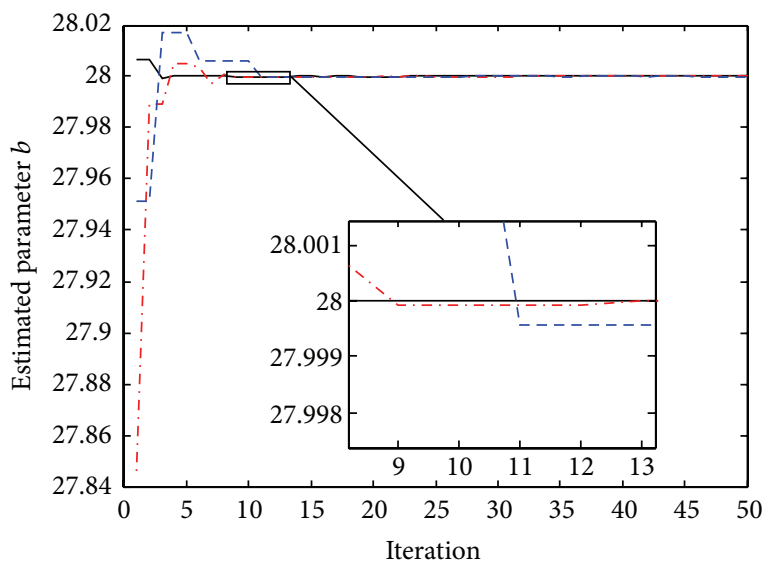

(b)

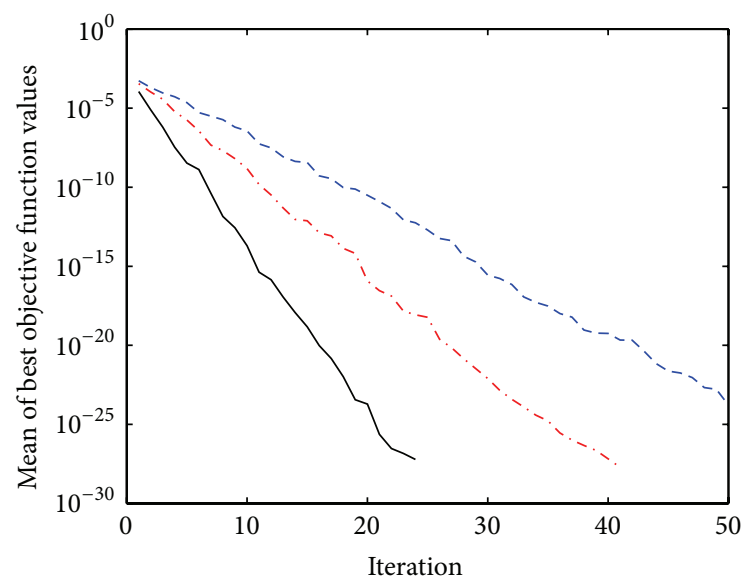

(d)

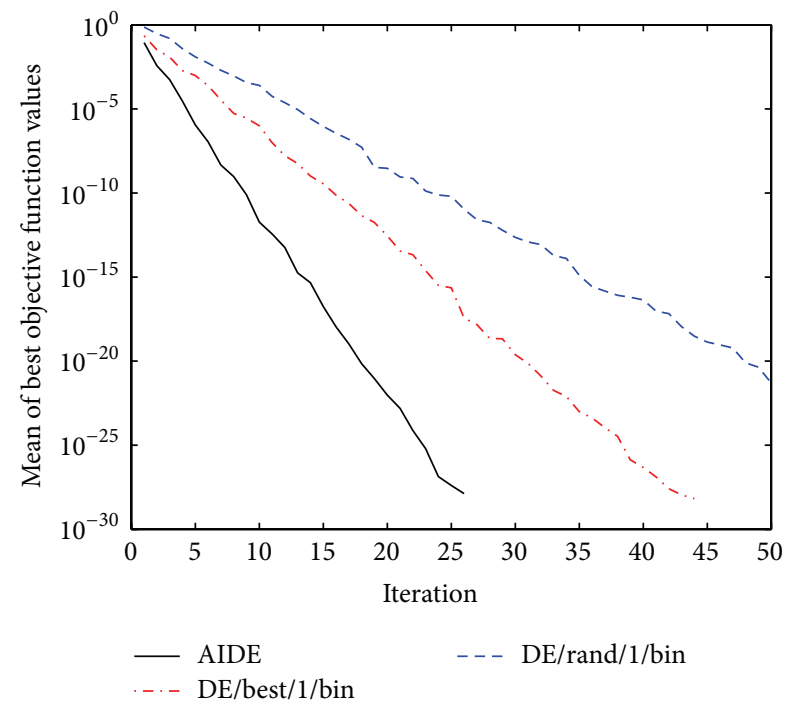

(f)

FIgURE 3: The evolution process of Lorenz system with one unknown parameter. 
TABLE 1: Statistical results of different approaches for one-dimensional parameter estimation.

\begin{tabular}{|c|c|c|c|c|}
\hline Approaches & Index & Best & Mean & Worst \\
\hline \multirow{2}{*}{ DE_rand } & $\widehat{a}$ & 10.000000000000009 & 9.9999999999999984 & 10.000000000001773 \\
\hline & $J$ & $2.2985 e-027$ & $1.6365 e-024$ & $1.6950 e-023$ \\
\hline \multirow{2}{*}{ DE_best } & $\widehat{a}$ & 10.000000000000000 & 10.000000000000000 & 10.000000000000000 \\
\hline & $J$ & 0.000000000000000 & 0.000000000000000 & 0.000000000000000 \\
\hline \multirow{2}{*}{ AIDE } & $\widehat{a}$ & 10.000000000000000 & 10.000000000000000 & 10.000000000000000 \\
\hline & $J$ & 0.000000000000000 & 0.000000000000000 & 0.000000000000000 \\
\hline \multirow{2}{*}{ DE_rand } & $\widehat{b}$ & 27.999999999999936 & 28.000000000005393 & 27.999999999965191 \\
\hline & $J$ & $1.3657 e-024$ & $1.0048 e-019$ & $5.8964 e-019$ \\
\hline \multirow{2}{*}{ DE_best } & $\widehat{b}$ & 28.000000000000000 & 28.000000000000000 & 28.000000000000000 \\
\hline & $J$ & 0.000000000000000 & 0.000000000000000 & 0.000000000000000 \\
\hline \multirow{2}{*}{ AIDE } & $\widehat{b}$ & 28.000000000000000 & 28.000000000000000 & 28.000000000000000 \\
\hline & $J$ & 0.000000000000000 & 0.000000000000000 & 0.000000000000000 \\
\hline \multirow{2}{*}{ DE_rand } & $\widehat{c}$ & 2.666666666666673 & 2.666666666666710 & 2.666666666666033 \\
\hline & $J$ & $4.4737 e-025$ & $4.9702 e-022$ & $3.7970 e-021$ \\
\hline \multirow{2}{*}{ DE_best } & $\widehat{c}$ & 2.6666666666666667 & 2.6666666666666667 & 2.6666666666666667 \\
\hline & $J$ & 0.000000000000000 & 0.000000000000000 & 0.000000000000000 \\
\hline \multirow{2}{*}{ AIDE } & $\widehat{c}$ & 2.666666666666667 & 2.6666666666666667 & 2.6666666666666667 \\
\hline & $J$ & 0.000000000000000 & 0.000000000000000 & 0.000000000000000 \\
\hline
\end{tabular}

DE_rand stands for DE/rand/1/bin and DE_best means DE/best/1/bin.

TABLE 2: Statistical results of different approaches for two-dimensional parameter identification ( $a$ and $b$ are unknown).

\begin{tabular}{lcccc}
\hline & Index & DE/rand/1/bin & DE/best/1/bin & AIDE \\
\hline \multirow{3}{*}{ Best } & $\widehat{a}$ & 9.999999999999080 & 10.000000000000000 & 10.000000000000000 \\
& $\widehat{b}$ & 28.000000000000142 & 28.000000000000000 & 28.000000000000000 \\
& $J$ & $8.4800 e-024$ & 0.000000000000000 & 0.000000000000000 \\
\hline \multirow{3}{*}{ Mean } & $\widehat{a}$ & 9.999999999992522 & 9.999999999999996 & 10.000000000000000 \\
& $\widehat{b}$ & 28.000000000000107 & 28.000000000000000 & 28.000000000000000 \\
& $J$ & $7.2633 e-021$ & $1.0373 e-028$ & 0.000000000000000 \\
\multirow{3}{*}{ Worst } & $\widehat{a}$ & 9.999999999950955 & 9.999999999999954 & 10.000000000000000 \\
& $\widehat{b}$ & 28.000000000009916 & 28.000000000000011 & 28.000000000000000 \\
& $J$ & $3.7208 e-020$ & $1.2561 e-027$ & 0.000000000000000 \\
\hline
\end{tabular}

TABLE 3: Statistical results of different approaches for two-dimensional parameter identification ( $a$ and $c$ are unknown).

\begin{tabular}{lcccr}
\hline & Index & DE/rand/1/bin & DE/best/1/bin & AIDE \\
\hline \multirow{3}{*}{ Best } & $\widehat{a}$ & 10.000000000000011 & 10.000000000000000 & 10.000000000000000 \\
& $\widehat{c}$ & 2.6666666666666698 & 2.66666666666666667 & 2.66666666666666667 \\
\multirow{3}{*}{ Mean } & $J$ & $8.8682 e-024$ & 0.000000000000000 & 0.000000000000000 \\
& $\widehat{a}$ & 9.999999999999748 & 10.000000000000011 & 10.000000000000000 \\
& $\widehat{c}$ & 2.666666666666668 & 2.666666666666665 & 2.666666666666667 \\
\multirow{3}{*}{ Worst } & $J$ & $3.8628 e-022$ & $2.0355 e-028$ & 0.000000000000000 \\
& $\widehat{a}$ & 10.000000000007114 & 10.000000000000023 & 10.000000000000000 \\
& $\widehat{c}$ & 2.666666666666092 & 2.666666666666663 & 2.666666666666667 \\
& $J$ & $3.7443 e-021$ & $5.6290 e-028$ & 0.000000000000000 \\
\hline
\end{tabular}

unknown parameters and objective values are illustrated in Figures 4-6, respectively.

From Tables 2-4, it can be seen that high-quality solutions are obtained by AIDE and their values are all equal to the corresponding true values. Although the best results found by $\mathrm{DE} /$ best/1/bin are also equal to the true values, the worst results obtained by DE/best/1/bin are all worse than those of AIDE for the three cases, which shows that sometimes $\mathrm{DE} /$ best/1/bin may result in premature convergence and
AIDE can achieve a good compromise between the exploration and the exploitation. In contrast, the performance of $\mathrm{DE} / \mathrm{rand} / 1 / \mathrm{bin}$ is the worst among the three methods. All these can also be confirmed from Figures 4-6, respectively.

\subsubsection{Simulation on Three-Dimensional Parameter Estima-} tion. In this section, three-dimensional parameter estimation of Lorenz system is considered. In the case, the three parameters $a, b$, and $c$ are all unknown and need to be identified. 
TABLE 4: Statistical results of different approaches for two-dimensional parameter identification ( $b$ and $c$ are unknown).

\begin{tabular}{lcccr}
\hline & Index & DE/rand/1/bin & DE/best/1/bin & AIDE \\
\hline \multirow{3}{*}{ Best } & $\widehat{b}$ & 27.999999999994451 & 28.000000000000000 & 28.000000000000000 \\
& $\widehat{c}$ & 2.666666666664585 & 2.666666666666667 & 2.666666666666667 \\
& $J$ & $8.7373 e-021$ & 0.000000000000000 & 0.000000000000000 \\
\hline \multirow{3}{*}{ Mean } & $\widehat{b}$ & 28.000000000004341 & 28.000000000000000 & 28.000000000000000 \\
& $\widehat{c}$ & 2.666666666667481 & 2.666666666666666 & 2.666666666666667 \\
& $J$ & $4.2924 e-019$ & $8.8037 e-029$ & 0.000000000000000 \\
\hline \multirow{3}{*}{ Worst } & $\widehat{b}$ & 28.000000000194063 & 28.000000000000000 & 28.000000000000000 \\
& $\widehat{c}$ & 2.666666666707930 & 2.666666666666666 & 2.666666666666667 \\
& $J$ & $1.6599 e-018$ & $8.8037 e-028$ & 0.000000000000000 \\
\hline
\end{tabular}
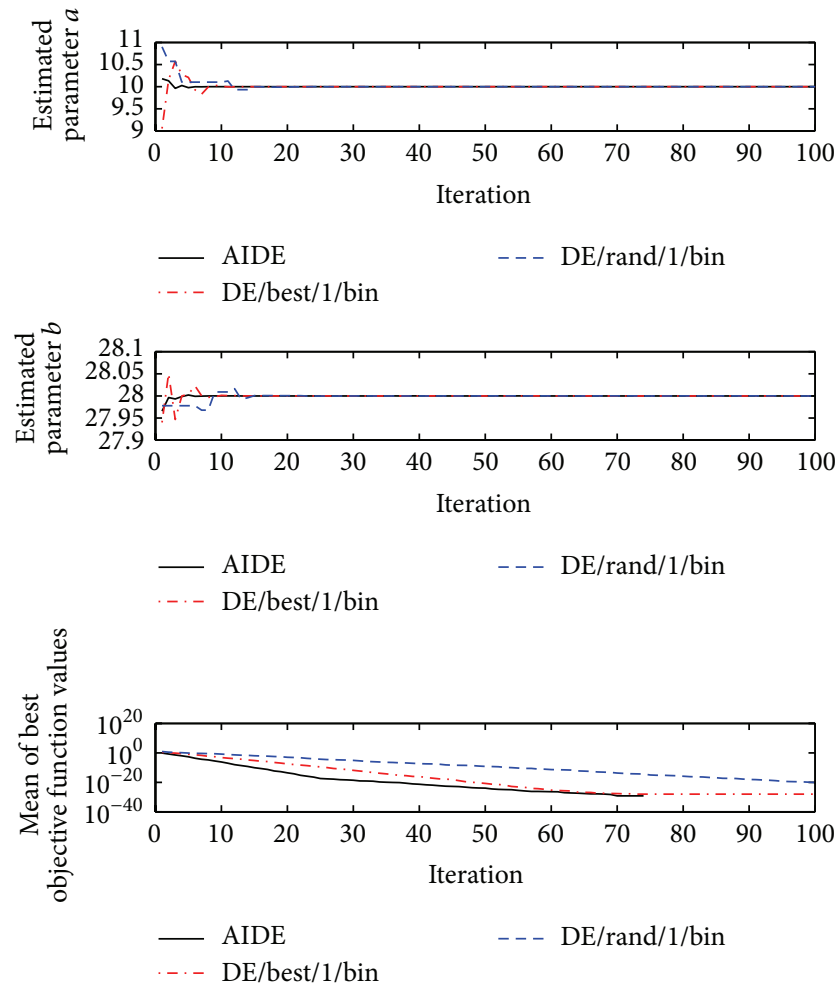

FIGURE 4: The evolution process of Lorenz system when parameters $a$ and $b$ are unknown.

The results are shown in Table 5 in terms of best, mean, and worst of the results obtained in 20 independent runs by each algorithm. The evolution processes of unknown parameters and objective value are illustrated in Figure 7, respectively.

As shown in Table 5, all the results obtained by AIDE are still equal to the true values. AIDE is superior to both DE/ rand/1/bin and DE/best/1/bin according to the mean value. Comparatively, DE/rand/1/bin is slightly better than DE/best/ $1 /$ bin in terms of the mean and worst values. Namely, as parameter dimension increases, the performance of DE/best/ $1 /$ bin is not consistently better than that of $\mathrm{DE} / \mathrm{rand} / \mathrm{1} / \mathrm{bin}$ as before. This may be the reason that the guidance of the global best individual leads to the premature convergence of $\mathrm{DE} /$ best/1/bin. Instead, DE/rand/1/bin obtains a good convergence performance as the iterative generation increases.
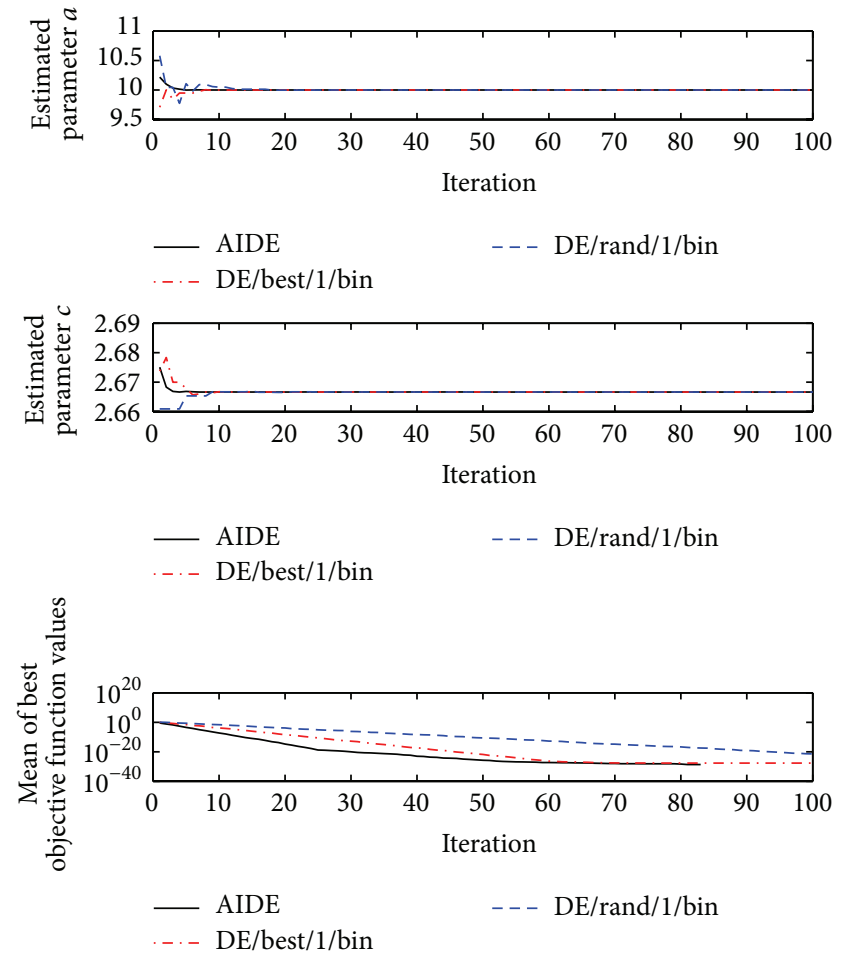

FIGURE 5: The evolution process of Lorenz system when parameters $a$ and $c$ are unknown.

All in all, AIDE is more effective and robust than other two algorithms, which can also be seen from Figure 7. All these also demonstrate that the modifications of AIDE are effective for balancing the exploration and exploitation ability of it.

5.2.4. Simulation on Lorenz System with Noise. Subsequently, the three-dimensional parameter estimation of Lorenz system with noise is considered again. As shown in Figure 1, each system state vector $\left(x_{1}, x_{2}, x_{3}\right)$ is added with white noise in the range of $[-0.1,0.1]$ in the experiment. Then AIDE was independently executed 20 times and the statistical results are given in Table 6 . The parameter settings of AIDE in the case are the same as the aforementioned in Section 5.1.

From Table 6, it can also be observed that AIDE achieves good results when noise exists. In addition, the relative 
TABle 5: Statistical results of different approaches for three-dimensional parameter identification ( $a, b$, and $c$ are unknown).

\begin{tabular}{ccccc}
\hline & Index & DE/rand/1/bin & DE/best/1/bin & AIDE \\
\hline \multirow{3}{*}{ Best } & $\widehat{a}$ & 10.000000000000000 & 10.000000000000000 & 10.000000000000000 \\
& $\widehat{b}$ & 28.000000000000000 & 28.000000000000000 & 28.000000000000000 \\
& $\widehat{c}$ & 2.666666666666667 & 2.666666666666667 & 2.666666666666667 \\
\multirow{3}{*}{ Mean } & $J$ & 0.000000000000000 & 0.000000000000000 & 0.000000000000000 \\
& $\widehat{a}$ & 10.000000000000000 & 9.999999999999996 & 10.000000000000000 \\
& $\widehat{b}$ & 28.000000000000000 & 28.000000000000000 & 28.000000000000000 \\
& $\widehat{c}$ & 2.666666666666666 & 2.666666666666666 & 2.666666666666667 \\
\multirow{3}{*}{ Worst } & $J$ & $2.6737 e-029$ & $2.7318 e-028$ & 0.000000000000000 \\
& $\widehat{a}$ & 10.000000000000007 & 9.999999999999941 & 10.000000000000000 \\
& $\widehat{b}$ & 27.999999999999996 & 28.000000000000014 & 28.000000000000000 \\
& $\widehat{c}$ & 2.666666666666665 & 2.666666666666674 & 2.666666666666667 \\
& $J$ & $1.4778 e-028$ & $2.2160 e-027$ & 0.000000000000000 \\
\hline
\end{tabular}

TABLE 6: Statistical results of AIDE for three-dimensional parameter identification of Lorenz system with noise.

\begin{tabular}{lcccc}
\hline Methods & Index & Best & Mean & Worst \\
\hline \multirow{4}{*}{ AIDE } & $\widehat{a}$ & 9.990801271058711 & 9.998131753034325 & 9.989687785636363 \\
& $\widehat{b}$ & 28.003818708942255 & 28.000608072179272 & 28.002414544105758 \\
& $\widehat{c}$ & 2.667512761713986 & 2.666799322681526 & 2.667073063185854 \\
& $J$ & 0.0077 & 0.0082 & 0.0085 \\
\hline
\end{tabular}
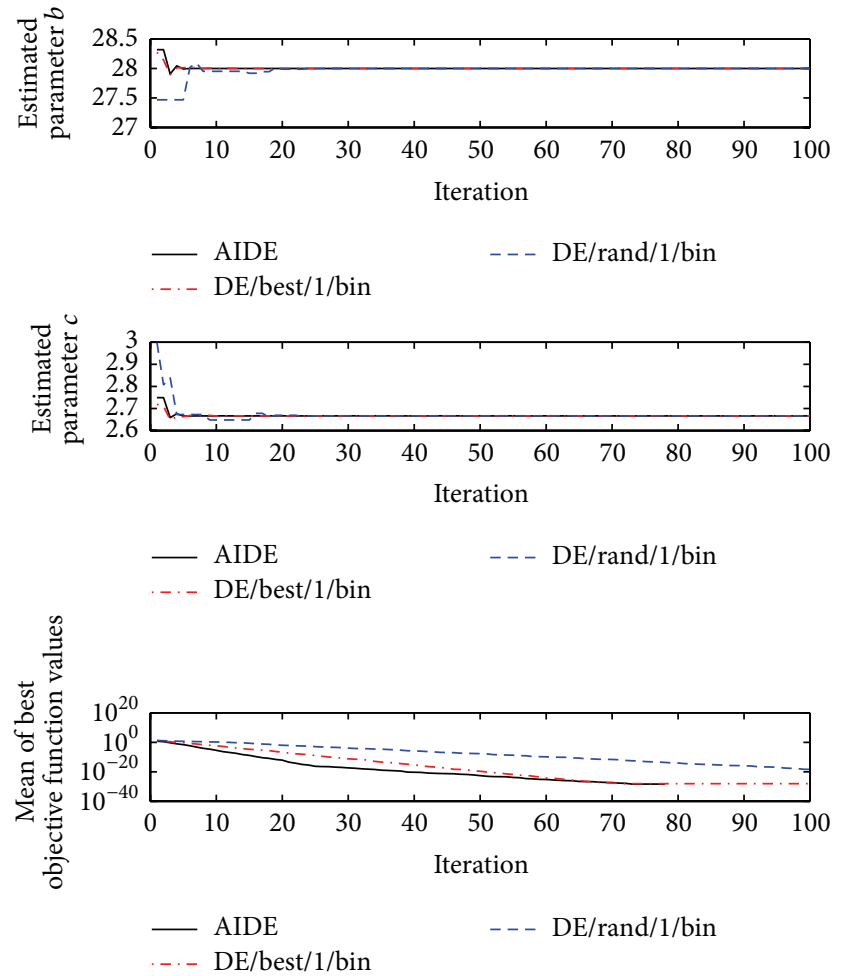

FIGURE 6: The evolution process of Lorenz system when parameters $b$ and $c$ are unknown.

errors between the estimated values and the true values of parameters $a, b$, and $c$ are approximately equal to $1 e-4,1 e-5$, and $1 e-5$, respectively, in terms of the mean values.
5.3. Comparison between GA, PSO, NMDE, and AIDE. In order to further validate the convergence performance of AIDE, it is compared with other well-known algorithms including genetic algorithm [19], particle swarm optimization [16], and NMDE [14] again.

In view of very good results obtained by NMDE, for convenience, three-dimensional parameter estimation is considered in this section. The parameter settings of AIDE are the same as the aforementioned. Namely, the population size is set to 120 and the maximum generation is set to 100 for GA, PSO, and NMDE, which is the same as those employed in the literatures $[14,16]$. Thus, there are about 300 generations in AIDE when the population size is fixed to 40 for a fair comparison. Next, the comparison results are listed in Table 7, where the results of GA, PSO, and NMDE are directly taken from the literatures $[14,16]$ for the sake of reliability.

From Table 7, it can be found that the best objective value obtained by NMDE is equal to that searched by AIDE, but the quality of best solutions $(a, b$, and $c)$ obtained by NMDE is worse than that of solutions obtained by AIDE. In particular, the worst results obtained by NMDE are obviously inferior to those found by AIDE. However, NMDE is obviously superior to both GA and PSO. Therefore, AIDE is the best algorithm among the four approaches for parameter identification of chaotic systems.

\section{Conclusion}

Through designing an appropriate objective function, parameter identification of chaotic systems is mathematically formulated as a global multidimensional optimization problem. Then, an alternate iterative differential algorithm, AIDE for short, is presented to solve the global optimization problem. 

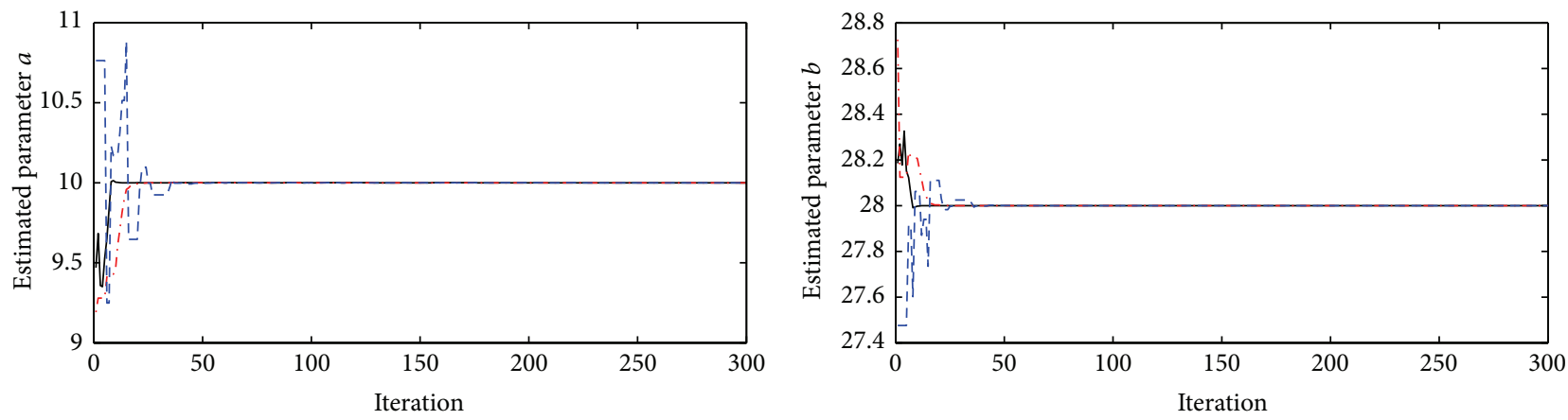

- AIDE

$---\mathrm{DE} / \mathrm{rand} / 1 / \mathrm{bin}$

- AIDE

- - $\mathrm{DE} / \mathrm{rand} / 1 / \mathrm{bin}$

-. - DE/best/1/bin

-. - DE/best/1/bin
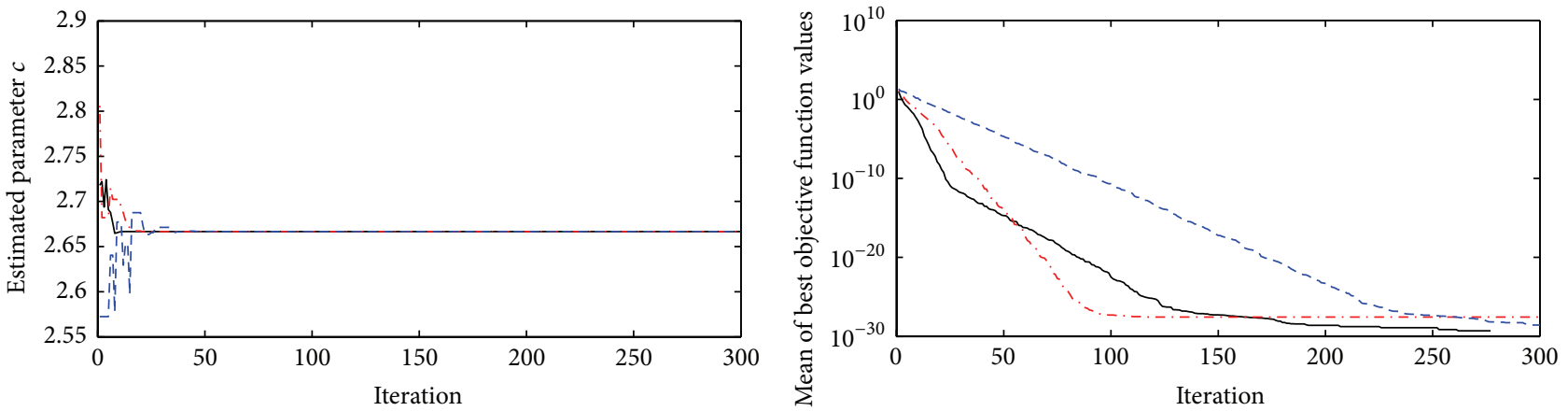

- AIDE
- - DE/best/1/bin

$---\mathrm{DE} / \mathrm{rand} / 1 / \mathrm{bin}$

- AIDE

- - $\mathrm{DE} / \mathrm{rand} / 1 / \mathrm{bin}$

FIGURE 7: The evolution process of Lorenz system when parameters $a, b$, and $c$ are unknown.

TABLE 7: Statistical results of different methods for three-dimensional parameter identification of Lorenz system.

\begin{tabular}{cccccc}
\hline & Index & GA & PSO & NMDE & AIDE \\
\hline \multirow{3}{*}{ Best } & $\widehat{a}$ & 10.067167 & 9.995332 & 10.000003 & $\mathbf{1 0 . 0 0 0 0 0 0 0 0 0 0 0 0 0 0 0}$ \\
& $\widehat{b}$ & 27.922058 & 28.007146 & 28.000002 & $\mathbf{2 8 . 0 0 0 0 0 0 0 0 0 0 0 0 0 0 0}$ \\
& $\widehat{c}$ & 2.663426 & 2.667013 & 2.666667 & $\mathbf{2 . 6 6 6 6 6 6 6 6 6 6 6 6 6 6 7}$ \\
\multirow{3}{*}{ Mean } & $J$ & 4.310715 & 0.048645 & 0.000000 & $\mathbf{0 . 0 0 0 0 0 0 0 0 0 0 0 0 0 0 0}$ \\
& $\widehat{a}$ & 10.139783 & 10.018417 & 10.000009 & $\mathbf{1 0 . 0 0 0 0 0 0 0 0 0 0 0 0 0 0 0}$ \\
& $\widehat{b}$ & 27.742735 & 27.993390 & 28.999980 & $\mathbf{2 8 . 0 0 0 0 0 0 0 0 0 0 0 0 0 0 0}$ \\
& $\widehat{c}$ & 2.648585 & 2.666281 & 2.666666 & $\mathbf{2 . 6 6 6 6 6 6 6 6 6 6 6 6 6 6 7}$ \\
\multirow{3}{*}{ Worst } & $J$ & 10.929003 & 10.608212 & 10.000223 & $\mathbf{0 . 0 0 0 0 0 0 0 0 0 0 0 0 0 0 0}$ \\
& $\widehat{a}$ & 26.127605 & 27.704424 & 27.999848 & $\mathbf{1 0 . 0 0 0 0 0 0 0 0 0 0 0 0 0 0 0}$ \\
& $\widehat{b}$ & 2.560249 & 2.657231 & 2.666660 & $\mathbf{2 8 . 0 0 0 0 0 0 0 0 0 0 0 0 0 0 0}$ \\
& $\widehat{c}$ & 6461.48006 & 39.406026 & $\mathbf{2 . 6 6 6 6 6 6 6 6 6 6 6 6 6 6 7}$ \\
\hline
\end{tabular}

Bold entities mean the best results.

First, a new mutation strategy $\mathrm{DE} /$ pbest/1 is proposed in AIDE. Second, the new mutation strategy and DE/best/1 are run alternately in AIDE. Meanwhile, a greedy selection mechanism is improved and employed. Last, a mutation mechanism like scout bees operation of artificial bee colony algorithm is introduced in order to better keep the population diversity. When compared with two variants of $\mathrm{DE}$, that is, $\mathrm{DE} / \mathrm{rand} / 1 /$ bin and $\mathrm{DE} / \mathrm{best} / \mathrm{l} / \mathrm{bin}$, the experimental results show that AIDE is better than both $\mathrm{DE} / \mathrm{rand} / 1 /$ bin and $\mathrm{DE} /$ best/1/bin, which means that AIDE is effective to achieve a compromise between the exploration and exploitation. Subsequently, a comparison between AIDE and other four famous metaheuristics is conducted. The comparison results demonstrate that AIDE outperforms GA, PSO, and NMDE. All these show that AIDE is an efficient and powerful tool for parameter identification of chaotic systems. 


\section{Conflict of Interests}

The authors declare that there is no conflict of interests regarding the publication of this paper.

\section{Acknowledgments}

This work is supported by the National Natural Science Foundation of China (Grant nos. 61263027 and 61364026) and the Science and Technology Foundation of Lanzhou Jiaotong University (Grant no. ZC2014010) and the New Teacher Project of Research Fund for the Doctoral Program of Higher Education of China (20126204120002) and the Natural Science Foundation of Gansu Province (Grant no. 148RJZA030).

\section{References}

[1] J. H. Lü, G. R. Chen, and S. C. Zhang, "A unified chaotic system and its research," Journal of the Graduate School of the Chinese Academy of Sciences, vol. 20, no. 1, pp. 123-129, 2003.

[2] W.-H. Ho, J.-H. Chou, and C.-Y. Guo, "Parameter identification of chaotic systems using improved differential evolution algorithm," Nonlinear Dynamics, vol. 61, no. 1-2, pp. 29-41, 2010.

[3] W.-D. Chang, "Parameter identification of Rossler's chaotic system by an evolutionary algorithm," Chaos, Solitons \& Fractals, vol. 29, no. 5, pp. 1047-1053, 2006.

[4] W.-D. Chang, "Parameter identification of Chen and Lü systems: a differential evolution approach," Chaos, Solitons and Fractals, vol. 32, no. 4, pp. 1469-1476, 2007.

[5] L. Li, Y. Yang, H. Peng, and X. Wang, "Parameters identification of chaotic systems via chaotic ant swarm," Chaos, Solitons \& Fractals, vol. 28, no. 5, pp. 1204-1211, 2006.

[6] J.-F. Chang, Y.-S. Yang, T.-L. Liao, and J.-J. Yan, "Parameter identification of chaotic systems using evolutionary programming approach," Expert Systems with Applications, vol. 35, no. 4, pp. 2074-2079, 2008.

[7] Y. Tang and X. Guan, "Parameter estimation for time-delay chaotic system by particle swarm optimization," Chaos, Solitons \& Fractals, vol. 40, no. 3, pp. 1391-1398, 2009.

[8] Y. Tang and X. Guan, "Parameter estimation of chaotic system with time-delay: a differential evolution approach," Chaos, Solitons \& Fractals, vol. 42, no. 5, pp. 3132-3139, 2009.

[9] L. Yuan, Q. Yang, and C. Zeng, "Chaos detection and parameter identification in fractional-order chaotic systems with delay," Nonlinear Dynamics, vol. 73, no. 1-2, pp. 439-448, 2013.

[10] H. Modares, A. Alfi, and M.-M. Fateh, "Parameter identification of chaotic dynamic systems through an improved particle swarm optimization," Expert Systems with Applications, vol. 37, no. 5, pp. 3714-3720, 2010.

[11] J. Sun, J. Zhao, X. Wu, W. Fang, Y. Cai, and W. Xu, "Parameter estimation for chaotic systems with a drift particle swarm optimization method," Physics Letters A, vol. 374, no. 28, pp. 28162822, 2010.

[12] L. Wang and L.-P. Li, "An effective hybrid quantum-inspired evolutionary algorithm for parameter estimation of chaotic systems," Expert Systems with Applications, vol. 37, no. 2, pp. 1279-1285, 2010.

[13] J.-P. Tien and T.-H. S. Li, "Hybrid Taguchi-chaos of multilevel immune and the artificial bee colony algorithm for parameter identification of chaotic systems," Computers \& Mathematics with Applications, vol. 64, no. 5, pp. 1108-1119, 2012.

[14] L. Wang, Y. Xu, and L. Li, "Parameter identification of chaotic systems by hybrid Nelder-Mead simplex search and differential evolution algorithm," Expert Systems with Applications, vol. 38, no. 4, pp. 3238-3245, 2011.

[15] J. Lü, G. Chen, D. Cheng, and S. Celikovsky, "Bridge the gap between the Lorenz system and the Chen system," International Journal of Bifurcation and Chaos, vol. 12, no. 12, pp. 2917-2926, 2002.

[16] Q. He, L. Wang, and B. Liu, "Parameter estimation for chaotic systems by particle swarm optimization," Chaos, Solitons \& Fractals, vol. 34, no. 2, pp. 654-661, 2007.

[17] J. Lü and G. Chen, "A new chaotic attractor coined," International Journal of Bifurcation and Chaos, vol. 12, no. 3, pp. 659661, 2002.

[18] Y. Yongguang and Z. Suochun, "Controlling uncertain Lü system using backstepping design," Chaos, Solitons \& Fractals, vol. 15, no. 5, pp. 897-902, 2003.

[19] D. Dai, X. K. Ma, F. C. Li, and Y. You, "An approach of parameter estimation for a chaotic system based on genetic algorithm," Acta Physica Sinica, vol. 51, no. 11, pp. 2459-2462, 2002 (Chinese).

[20] F. Gao and H.-Q. Tong, "Parameter estimation for chaotic system based on particle swarm optimization," Acta Physica Sinica, vol. 55, no. 2, pp. 577-582, 2006 (Chinese).

[21] L. Wang and Y. Xu, "An effective hybrid biogeography-based optimization algorithm for parameter estimation of chaotic systems," Expert Systems with Applications, vol. 38, no. 12, pp. 15103-15109, 2011.

[22] B. Peng, B. Liu, F.-Y. Zhang, and L. Wang, "Differential evolution algorithm-based parameter estimation for chaotic systems," Chaos, Solitons \& Fractals, vol. 39, no. 5, pp. 2110-2118, 2009.

[23] R. Storn and K. Price, "Differential evolution-a simple and efficient adaptive scheme for global opitmization," Tech. Rep. TR-95-012, International Computer Science Institute, Berkeley, Calif, USA, 1995.

[24] R. Storn and K. Price, "Differential evolution-a simple and efficient heuristic for global optimization over continuous spaces," Journal of Global Optimization, vol. 11, no. 4, pp. 341359, 1997.

[25] S. Rahnamayan, Opposition-based differential evolution [Ph.D. thesis], University of Waterloo, 2007.

[26] R. S. Rahnamayan, H. R. Tizhoosh, and M. M. A. Salama, "Opposition-based differential evolution," IEEE Transactions on Evolutionary Computation, vol. 12, no. 1, pp. 64-79, 2008.

[27] Z. W. Geem, J. H. Kim, and G. V. Loganathan, "A new heuristic optimization algorithm: harmony search," Simulation, vol. 76, no. 2, pp. 60-68, 2001.

[28] M. G. H. Omran and M. Mahdavi, "Global-best harmony search," Applied Mathematics and Computation, vol. 198, no. 2, pp. 643-656, 2008.

[29] D. Karaboga and B. Basturk, "A powerful and efficient algorithm for numerical function optimization: artificial bee colony (ABC) algorithm," Journal of Global Optimization, vol. 39, no. 3, pp. 459-471, 2007. 


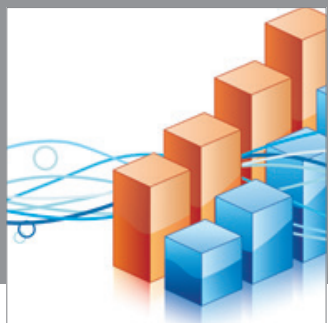

Advances in

Operations Research

mansans

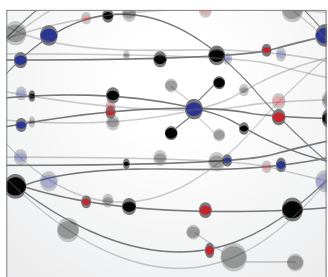

The Scientific World Journal
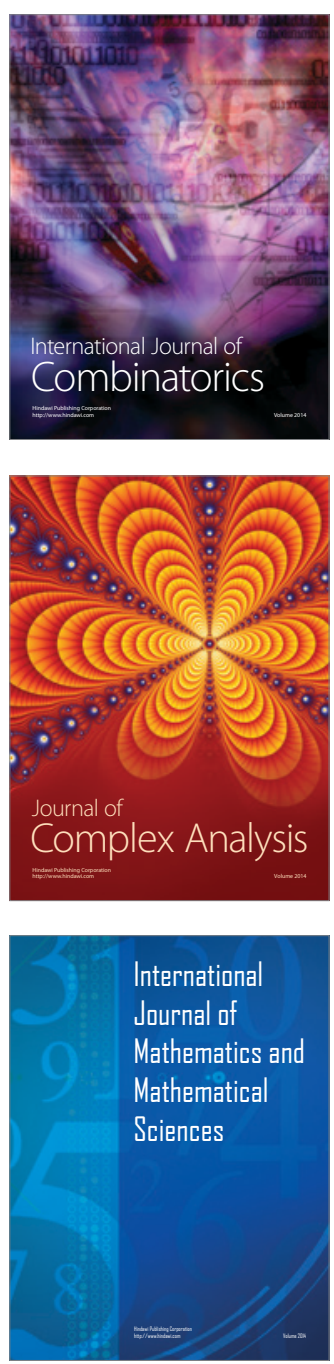
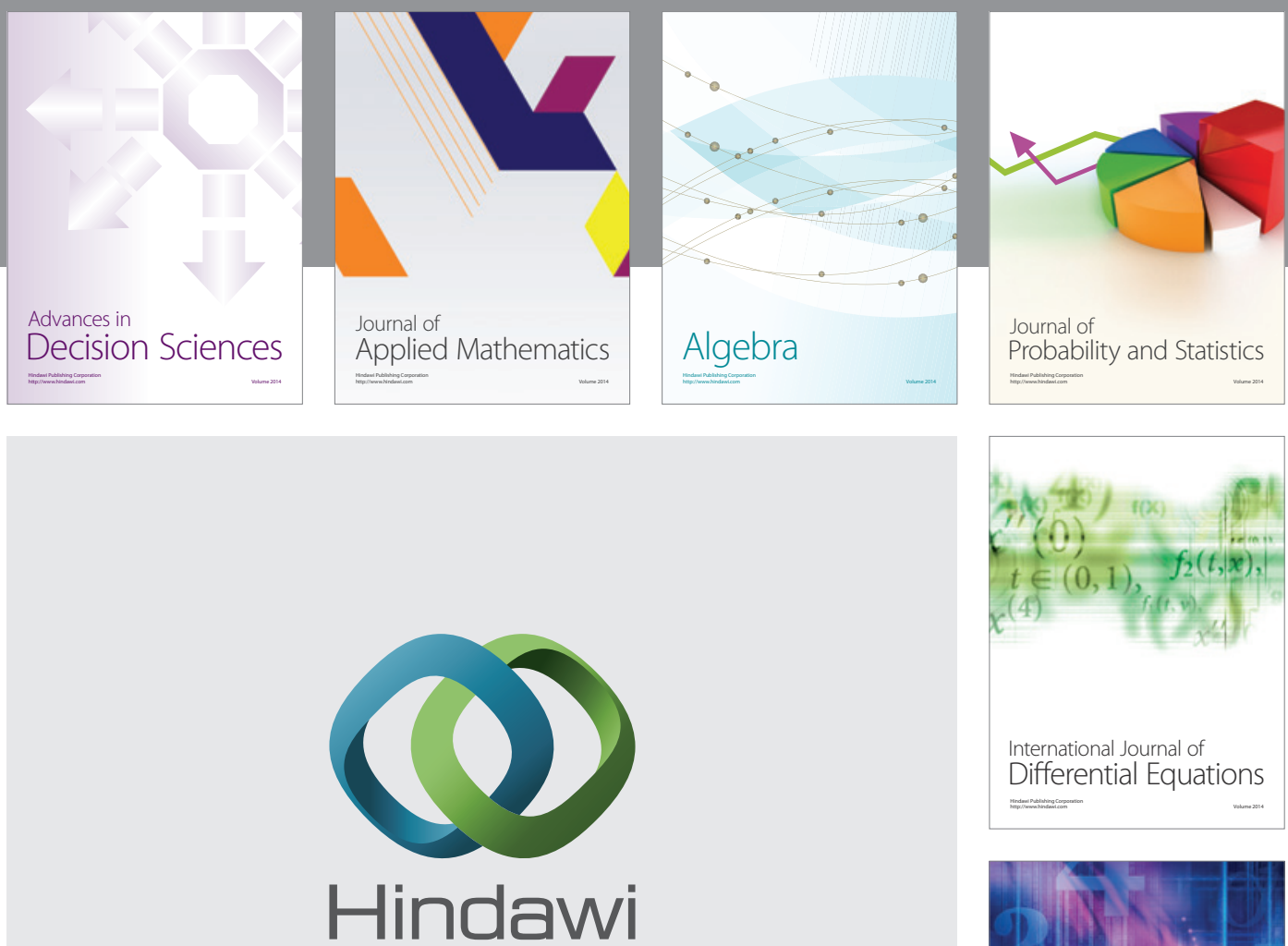

Submit your manuscripts at http://www.hindawi.com
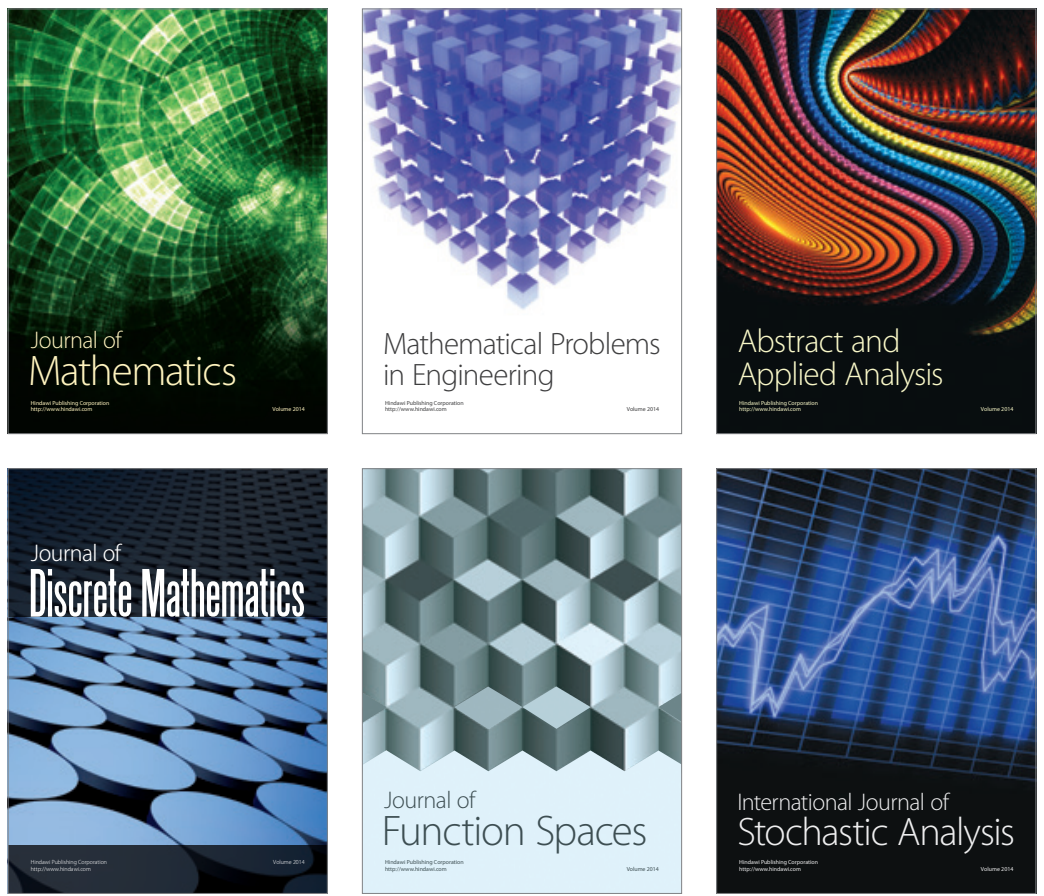

Journal of

Function Spaces

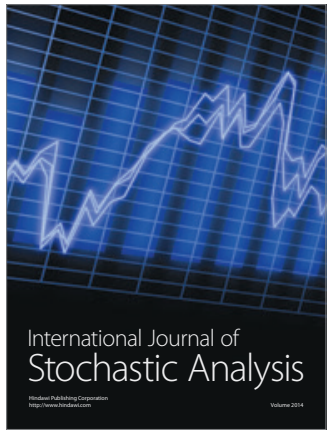

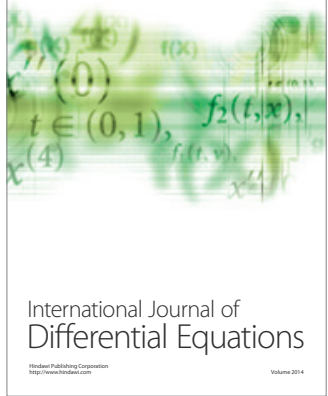
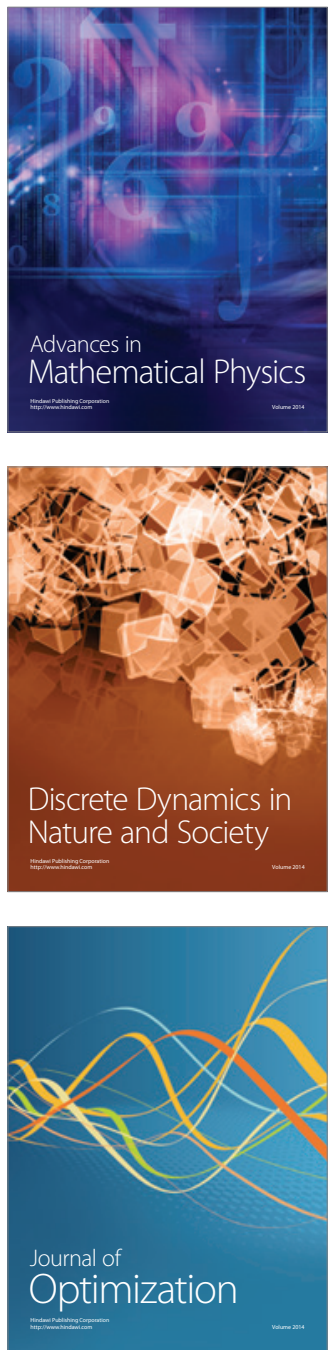